\title{
EVOLUCIÓN HISTÓRICA DEL MANTENIMIENTO INDUSTRIAL EN RELACIÓN A LA GESTIÓN DEL CONOCIMIENTO
}

口nu

Francisco-Javier Cárcel-Carrasco

Universitat Politècnica de València (España)

\section{DOI: http://dx.doi.org/10.6036/7890}

\section{INTRODUCCIÓN}

En los recursos de la ingeniería del mantenimiento industrial, son precisos equipos de medición muy especializados y un componente humano con alta especialización. Todo ello conlleva un fuerte conocimiento que debe ser gestionado en sus fases de creación, difusión y utilización. Los indicadores (normalmente utilizados) de rendimiento de mantenimiento se sustentan en diversos pilares como son la fiabilidad, disponibilidad, mantenibilidad, seguridad, los costes operativos, y uno fundamental para la operatividad como es el factor humano [1], dado el elevado conocimiento tácito que se genera en el desempeño de las funciones asignadas.

El conocimiento es la capacidad de actuar, procesar e interpretar información para generar más conocimiento o dar solución a un determinado problema. En este nuevo siglo se ha producido un cambio fundamental, en que el crecimiento de muchos sectores y de las empresas se ve impulsado por el conocimiento y las ideas, más, que teniendo en cuenta sólo por los recursos tradicionales [2]. Esto nos impulsa a una gestión estratégica de las empresas impulsada por el conocimiento, donde los activos tangibles tradicionales están perdiendo valor a favor de los intangibles $[3,4]$. Es por ello que se puede considerar el conocimiento como el principal ingrediente intangible tanto en las empresas como en la economía en su conjunto [5], y en la evolución del mantenimiento donde el peso del componente humano toma una gran incidencia, es un valor que ha tomado gran importancia con el avance de las técnicas y los recursos destinados a su mejora.

A la hora de plantear un servicio de mantenimiento, es de vital importancia tener un profundo conocimiento de las instalaciones, transformar el conocimiento tácito estratégico de las experiencias operativas de los operarios de mantenimiento en explícito, que sin duda profundizan en el estudio de las medidas de eficiencia energética y valorar la fiabilidad de las instalaciones, con el conocimiento del proceso del fallo, que hace mejorar la productividad de la empresa $[6,7]$ identificando los datos y la información relevante para mejorar el servicio $[8,9]$.

Con un cambio hacia un modelo basado en el Conocimiento y el Aprendizaje, la organización se centra en la capacidad de innovar y aprender, para resolver de una manera más eficiente sus trabajos cotidianos, así como resolver acciones nuevas o no rutinarias, creando un valor de lo intangible en base al conocimiento y a su rápida actualización en el ámbito del entorno de trabajo de la organización de mantenimiento. Debe ser asumido como una estrategia de desarrollo a largo plazo, visualizando el conocimiento como factor estratégico, así como una herramienta para la resolución de problemas y las tomas de decisiones [10].

En este artículo se pretende hacer una breve revisión de la evolución del mantenimiento para definir los elementos básicos que definen su naturaleza, y marcar su relación a los procesos ligados al conocimiento, para con ello, entender las tendencias futuras de la gestión de un intangible tan importante en dichos departamentos, cuya operativa incide de manera exponencial en la productividad de cualquier empresa.

\section{ELCONOCIMIENTO Y EL MANTENIMIENTO INDUSTRIAL}

En numerosa literatura existente se define que el conocimiento es poder, pero como lo afirma Nonaka [11], lo importante del conocimiento en las organizaciones depende de lo que se pueda hacer con él dentro de un ámbito de negocios. Es decir, el conocimiento por sí mismo no es relevante, en tanto no pueda ser utilizado para dar origen a acciones de creación de valor [12].

La gestión de conocimientos implica, por tanto, el uso de prácticas difíciles de observar y manipular, que a veces, son incluso desconocidas para los que las poseen. Esto presenta un problema para las empresas, más familiarizadas con la gestión y contabilidad del capital fijo. Entre las diversas categorías de inversiones relacionadas con el conocimiento (educación, formación, software, I+D, etc.), la gestión de conocimientos es una de las menos conocidas tanto cualitativa como cuantitativamente, así como en términos de costos y retornos económicos [5].

Son numerosos los factores del conocimiento y la experiencia que inciden en el mantenimiento (Figura 1), que basculan desde la propia misión a realizar y alcanzar las metas requeridas, innovar o mejorar el futuro de la actividad.

\section{LOS FACTORES DEL CONOCIMIENTO Y LA EXPERIENCIA EN MANTENIMIENTO}

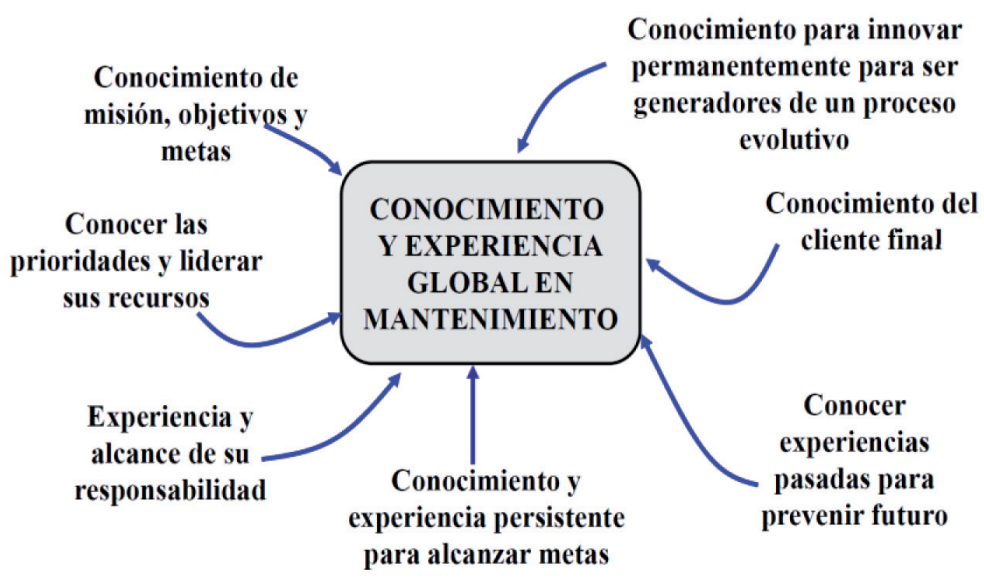

Fig. 1: Los factores del conocimiento en relación al mantenimiento. Fuente: Elaboración propia 
En la generación del conocimiento se produce una transformación del conocimiento tácito de los individuos en explícito a nivel grupal y organizativo [13], y cada uno de los miembros de tales colectivos lo interiorizan, convirtiéndolo de nuevo en tácito. Dicho proceso genera cuatro fases, que son: la socialización, externalización, combinación e internalización (figura 2), que pasan por cuatro fases fundamentales.

1. Socialización (de tácito a tácito): Es un proceso en el que se adquiere conocimiento tácito de otros, compartiendo experiencias y pensamientos con ellos, y comunicando ambos, de manera que quien los recibe incrementa su saber y llega a conseguir niveles cercanos a los del emisor. Este es un proceso de gran incidencia en los oficios industriales, especialmente en las labores de mantenimiento industrial: Existe una fase de acoplamiento en que el nuevo operario debe asimilar todo el equipamiento, equipos e instalaciones de la planta industrial, normalmente mediante comentarios y explicaciones de compañeros con más antigüedad. Es un proceso que en grandes plantas industriales puede durar meses o años en ser totalmente operativo el operario. do de equipos, etc), quedan explicitadas con breves partes de trabajo con indicación de la experiencia, quedando gran parte del conocimiento generado en forma tácita sólo en los miembros que han intervenido en la reparación o maniobra.

3. Combinación (de explícito a explícito): es la parte del proceso que sintetiza los conceptos explícitos y los traslada a una base de conocimiento, mediante los siguientes procedimientos [11]: captación e integración de nuevo conocimiento explícito esencial, a través de la recopilación, reflexión y síntesis; diseminación del mismo empleando los procesos de transferencia utilizados normalmente en la organización, tales como presentaciones, reuniones o correos electrónicos; y procesado, en documentos, planos, informes y datos de mercado. Se produce en los oficios industriales a través de la propia información técnica de la planta (planos de montaje, manuales de equipamiento, revisión de normativas, etc.), así como consulta de otras informaciones procesadas (históricos de mantenimiento, maniobras de las instalaciones, etc.)

4. Internalización (de explícito a tácito): es la etapa del proceso en la que se amplía el conocimiento tácito de los indivi-

\section{EL CONOCIMIENTO EN MANTENIMIENTO INDUSTRIAL SEGÚN MODELO NONAKA}

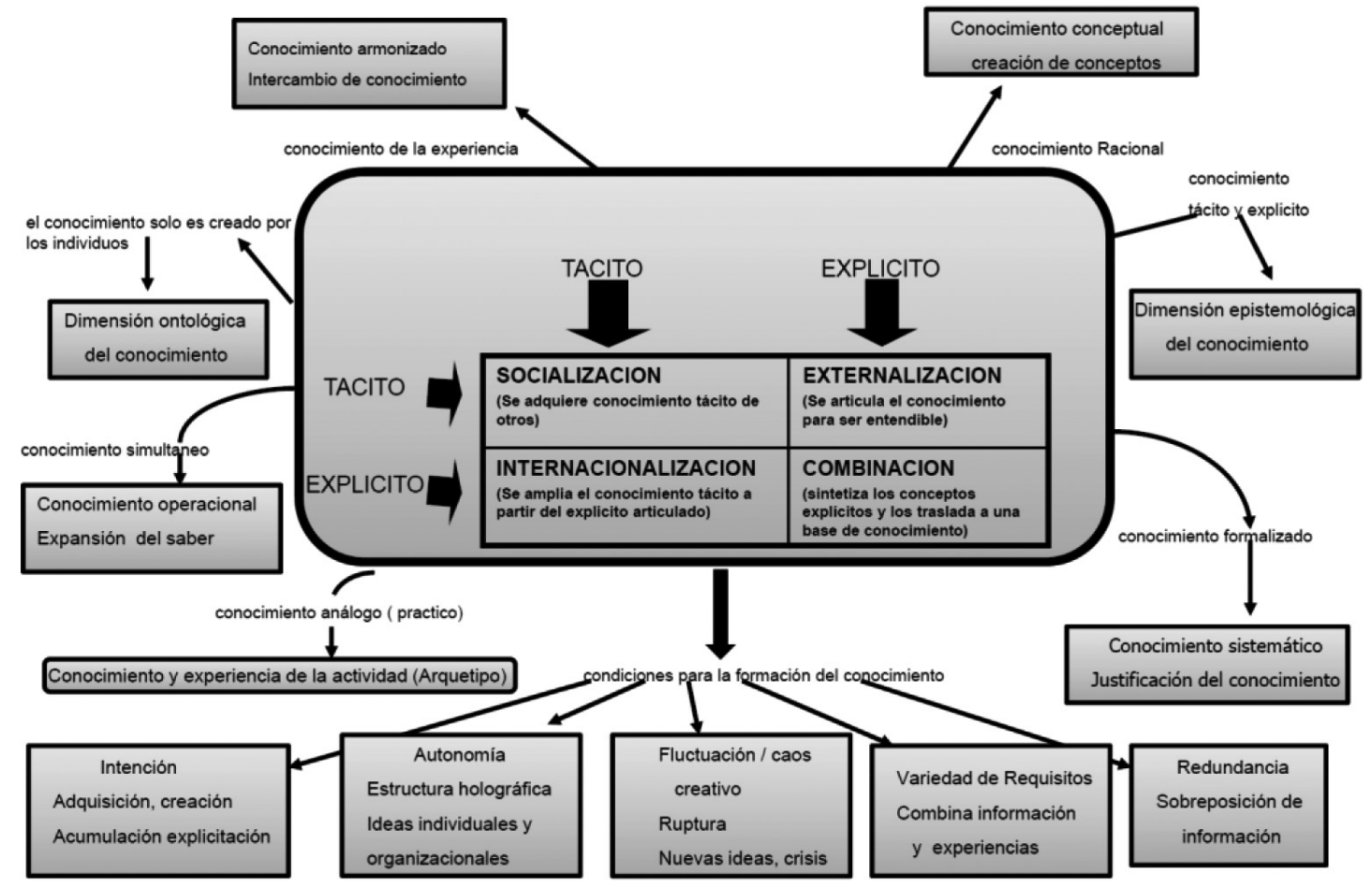

Fig. 2: El conocimiento industrial según modelo de Nonaka. Fuente: Elaboración propia a partir de [11,13]

2. Externalización (de tácito a explícito): etapa en la que se transforma el conocimiento tácito en conceptos explícitos o comprensibles para la organización o para cualquier individuo, a través de la propia articulación de éste y de su traslado a soportes rápidamente entendibles [11]. En los oficios industriales, esta etapa suele estar parcialmente sesgada. Se especifican partes de trabajo, tiempos de ejecución o periodos de realización de los trabajos, pero normalmente, las experiencias más valiosas (descripción de la reparación de una avería crítica, maniobras de emergencia, rearma- duos a partir del conocimiento explícito de la organización, al depurarse este último y convertirse en conocimiento propio de cada persona. Dicha internalización requiere por un lado, la actualización de los conceptos o métodos explícitos $y$, por otro, la inclusión de dicho conocimiento explícito en tácito [11]. Es necesario que el conocimiento explícito sea vivido o experimentado, bien pasando personalmente por la experiencia de realizar una actividad, o bien a través de la participación, para que así el individuo lo internalice según su propio estilo y hábitos. De esta forma los individuos usarán esta etapa para ampliar, extender y transformar su 
propio conocimiento tácito iniciando de nuevo el ciclo. En las actividades tácticas de mantenimiento o explotación, se produce a través de la propia información técnica de la planta, que los operarios deben asimilar para realizar los trabajos especificados.

\section{EVOLUCIÓN HISTÓRICA DEL MANTENIMIENTO Y SU RELACIÓN CON EL CONOCIMIENTO}

De forma muy sucinta, se realiza a continuación una breve reseña de la evolución del mantenimiento, a efectos de determinar la línea básica de esa evolución, extrayendo los elementos comunes a la misma y certificando la necesidad perentoria de procesos ligados al conocimiento, como algo imprescindible y valioso de la actividad de mantenimiento.

El mantenimiento en su desempeño óptimo suele pasar por tres operativas básicas, pasando desde la inspección, hacia las acciones de conservación así como las labores y experiencias de reparación, que conllevan estados de conocimiento que deben sintetizarse. Aunque es cierto que las operativas citadas son comunes en el día a día de una planta, no es menos cierto que el hecho de que se lleven a cabo depende de la política de mantenimiento fijada por el responsable correspondiente. Además, el hecho de llevarlas a cabo no implica que el mantenimiento sea óptimo ni que se recoja adecuadamente el conocimiento generado.

Aunque el origen del mantenimiento es, sin duda, tan antiguo como las primeras máquinas que utilizó el hombre, el mantenimiento industrial tal como lo entendemos, hizo su aparición, como actividad sistemáticamente organizada, en los albores del siglo XX. Tuvieron lugar, al parecer, los primeros casos conocidos, en fundiciones de Estados Unidos, y en el sector militar: en aviones y submarinos durante la Primera Guerra Mundial. En 1920, el mantenimiento mecánico ya se practicaba en plantas industriales, actividades de transporte, etc. De 1928 a 1930, aparecen las primeras empresas consultoras en este ámbito. No es casual que en fecha tan temprana aparezcan asesores cuyo producto es un servicio basado en su experiencia y conocimientos.

Con posterioridad a esta etapa inicial, el mantenimiento, como otros campos de la Organización Industrial, experimentan un notable desarrollo durante la Segunda Guerra Mundial y en la posguerra, en diferentes aplicaciones de interés militar. Se desarrollan programas de mantenimiento preventivo consistentes en inspeccionar el avión antes de cada vuelo, comprobando su estado y reemplazando componentes después de un cierto número de horas de funcionamiento (técnica, también empleada en la actualidad, conocida como "hard time"). Se revelan, así, los conocimientos sobre el estado de los equipos y la rentabilidad del reemplazo, como algo básico para proceder eficientemente a la conservación de los mismos.

A partir de 1945, se utiliza la redundancia de componentes en el diseño, reduciendo la "criticidad del fallo". También se formalizan técnicas de ensayo y medidas físicas, con el fin de conocer la probabilidad de fallo de cada componente. Comienza a "protocolizarse" la actividad de mantenimiento en lo relativo a pruebas y ensayos, con lo que se obtiene una información registrada y formalizada, objeto, por tanto, de conocimiento explícito. La medición introduce el método científico riguroso encaminado al conocimiento del elemento más valioso y sustancial del objeto del mantenimiento: el fallo y sus características.

A finales de la década de los 60, comienza la aplicación sis- temática de las técnicas de fiabilidad, permitiendo la predicción de los costes derivados de los fallos y el cálculo de la rentabilidad de la actividad de mantenimiento. Aun así, el comportamiento de determinados componentes no presentaba fácil predicción, escapándose de los modelos al uso; algo que aún hoy perdura, ya que se pasa, del modelo clásico de curva de tasa de fallo en forma de bañera, a seis modelos diferentes en el mantenimiento basado en la fiabilidad (RCM), y la investigación continúa tratando de modelar aquellos comportamientos que no se adaptan suficientemente a los esquemas planteados. El conocimiento sobre el comportamiento del fallo, a lo largo del ciclo de vida de los equipos, se revela como complejo y específico y es de esperar que, en adelante, se planteen modelos multidisciplinares que aborden la relación causa-efecto del fallo en toda su complejidad, esto es, entendiendo la máquina o el equipo como un todo.

Además de la atención al análisis y estudio de las causas y efectos de las incidencias de los equipos industriales, objeto básico del mantenimiento, también su gestión fue ya desde el comienzo de la década de los 60 elemento de estudio y análisis, Así desde el comienzo de los años 60, se destaca la utilidad de disponer de estadísticas históricas de averias para el análisis y planificación del mantenimiento $[14,15]$, así como la descripción de diversos métodos de optimización de políticas de mantenimiento [16]. En esa línea, pero fijando como objetivo el aumento de productividad, Darnell y Bert en 1978 [17], publican una contribución en la que abogan por el mantenimiento programado como medio de aumentar la productividad [17]. Christer, en 1981 y Boland y Proscan en 1982, insisten en la misma línea, destacando la incidencia sobre la productividad de una actitud activa y programada en mantenimiento, en contraposición con actitudes pasivas e incontroladas $[18,19]$.

La información ya de por sí compleja y específica, en el análisis de causas, se hace ahora desbordante por causa de los múltiples aspectos de interés y su relación. El tratamiento de la información exige técnicas informáticas y de Investigación Operativa depuradas que den sentido a esa información, la orienten a las metas propuestas en la planificación y dirijan su uso a la optimización de la actividad de mantenimiento.

Aunque es a finales de la década de los 60 cuando las asociaciones de mantenimiento impulsaron los estudios, correspondientes a esta disciplina, especialmente en Inglaterra [20], es en los 70 cuando aparecen claramente dos líneas de análisis que configuran dos escuelas de pensamiento acerca del Mantenimiento.

Una línea de análisis, la Escuela Soviética, contempla los parámetros del mantenimiento de máquinas desde un punto de vista constructivo y biológico, introduciendo conceptos de salud y envejecimiento. Artobolevski y Gorvachkin destacan la relación de los parámetros de estado en el diseño de máquinas; haciendo éste último referencia, en sus análisis, al modelado del desgaste de la máquina, e incorporando al diseño especificaciones orientadas a la facilidad con que se efectúe la reparación. De destacar también son los trabajos de Artemieb y Raiman sobre obtención y análisis de datos en el proceso de desgaste de los tractores. Finalmente, Selivanov introdujo en 1972 la noción de utilidad como una variable o característica objetivo del servicio de las máquinas.

Es a partir de esa época cuando comienza a verse con mayor necesidad en clasificar los tipos de mantenimiento, introduciéndose en mayor medida el mantenimiento planificado, con una proactividad que daría lugar a las diferentes técnicas organizativas del mantenimiento industrial que evolucionan hasta nuestros 
días, marcando la necesidad de un mayor grado de transferencia y gestión del conocimiento en todas las actividades implicadas y que implica de manera profunda al componente humano, tan intensivo en esta actividad. Una mayor definición de la clasificación del mantenimiento (Figura 3) se puede encontrar en la norma UNE EN 13306 donde se especifica definiciones y términos genéricos para áreas técnicas, administrativas y gerenciales del mantenimiento.

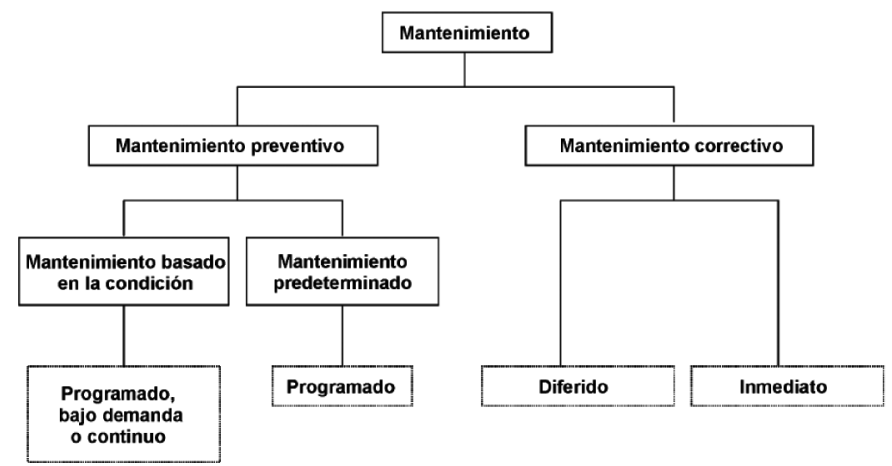

Fig. 3: Clasificación general del mantenimiento según UNE-EN 13306. Fuente: UNE EN 13306

Por otro lado, la escuela occidental contempla, dentro del estudio del mantenimiento, los conceptos económicos básicos de la Teoría Económica de la Firma, para determinar la rentabilidad de la reparación, y en consecuencia la fijación de políticas de mantenimiento, en relación, por ejemplo, con la renovación de la maquinaria. A este enfoque que puede ser calificado como clásico, a partir de 1975 se introduce el de la terotecnología, que contempla el estudio del coste del ciclo de vida (LCC o "life cycle costing"), como necesario a la hora de concluir estrategias de inversión, operación y eliminación en mantenimiento [21].

Ambos enfoques se integran modernamente en un enfoque de sistemas en que la conservación no puede deslindarse del diseño y la logística a lo largo de la vida del equipo y la planta (figura 4). Los conocimientos en este campo se interrelacionan con el resto de información y experiencia de los procesos de producción y distribución. El conocimiento integrado toma carta de valor y aparece como imprescindible, si lo que se busca son objetivos

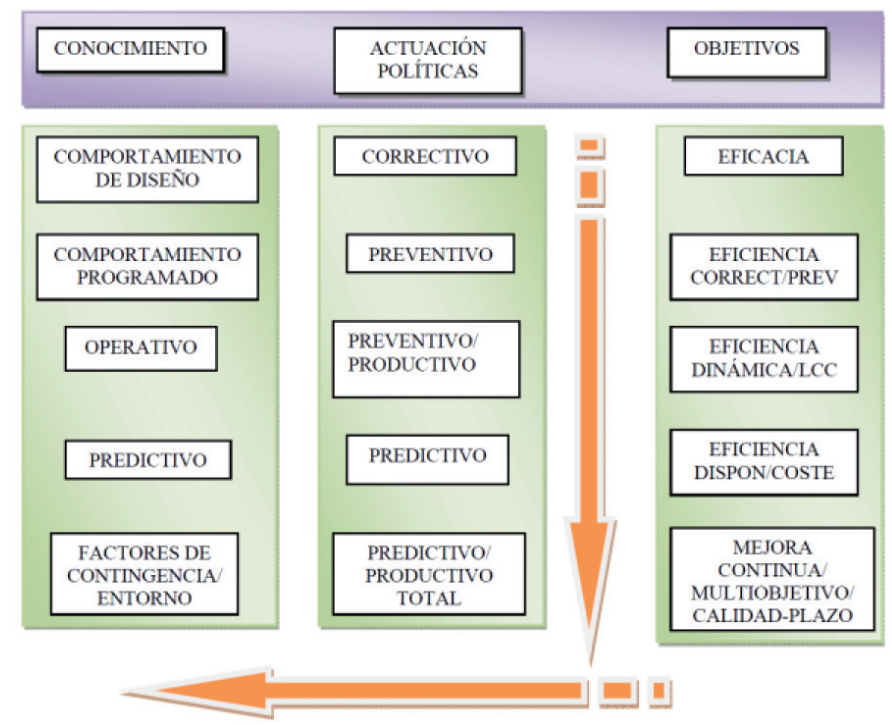

Fig. 4: Esquema general de la evolución del mantenimiento. Fuente: Elaboración propia de sostenibilidad y productividad compartidas en los proyectos industriales.

También la actividad de mantenimiento se ha contemplado desde un punto de vista macroeconómico o sectorial, así, en 1970, el Ministerio de Tecnología del Reino Unido publica un informe sobre la incidencia del mantenimiento en la economía nacional e incluso, con posterioridad, en 1977, presenta previsiones para el final de la década [22]. Las previsiones auguraban un notable incremento de la actividad de mantenimiento y paralelamente un aumento de costes, acorde con los datos procedentes de la economía americana, en el sentido de que las plantas más modernas tienen un mantenimiento más caro. Este informe del partido laborista británico acerca de la situación en la industria, contemplaba ya la actividad de mantenimiento separada de la de producción.

Este enfoque sistémico se recoge en el documento "Maintenance Steering Group", también conocido como MSG-2, fruto de las deliberaciones de un comité impulsado por Boeing y Pratt and Whitney. En él se identificaban los sistemas del avión; dentro de ellos, subsistemas, y así hasta llegar a las unidades críticas, empleando criterios de seguridad o economía [44-45]. A partir de las unidades críticas se definían las funciones individuales para cada una de ellas. Finalmente, para cada función, se especificaban los posibles modos de fallo y a partir de ellos se establecian las políticas de mantenimiento.

Con la explosión de las nuevas tecnologías de la información, el tratamiento del conocimiento en general, y en concreto el obtenido en la planta industrial, ha cobrado una nueva dimensión, apareciendo como el activo más valioso en escenarios futuros [23-25].

Los efectos de la automatización están posibilitando una monitorización en tiempo real del mantenimiento [26]. Así por ejemplo, determinados sistemas, como algunas aeronaves, incorporan ya monitorizaciones de estado que analizan los datos de las unidades en servicio y especifican las acciones a tomar. Son previsibles fuertes sinergias en el uso combinado de las nuevas tecnologías de la información y las comunicaciones y las tecnologías propias del mantenimiento (predictivo, auto-diagnóstico, mantenimiento a distancia, sistemas de mantenimiento a través de internet, etc.).

Es especialmente a partir de la década de los años 70, 80 y 90 del siglo XX, cuando se intensifica la utilización de estrategias organizativas de mantenimiento, aunque normalmente en el entorno de grandes empresas, como el basado en la fiabilidad (RCM) [27,28], el mantenimiento productivo total (TPM) [29], el mantenimiento efectivo [41,30], proactivo [31,32], reactivo [33], de clase mundial WCM [34], mantenimiento centrado en el riesgo [35], así como otros muchos modelos teóricos. Hay que tener en cuenta, el nivel estratégico de dicha actividad, con gran dependencia sobre las áreas de producción o servicios [23], así como el estudio profundo de fiabilidad de todos los componentes intervinientes [36-38]. En la figura 5 se observan las tendencias en la gestión de los servicios de mantenimientos en los que se observa la necesidad de mayor incidencia de la información y el conocimiento para una práctica eficiente y económica de dicho servicio.

Es importante aclarar que no todas las empresas evolucionan históricamente al pasar por cada una de las tácticas en forma secuencial, simplemente adoptan una propia que reúne las mejores prácticas de varias de ellas, para recalcar que el TPM es la más básica de todas. 


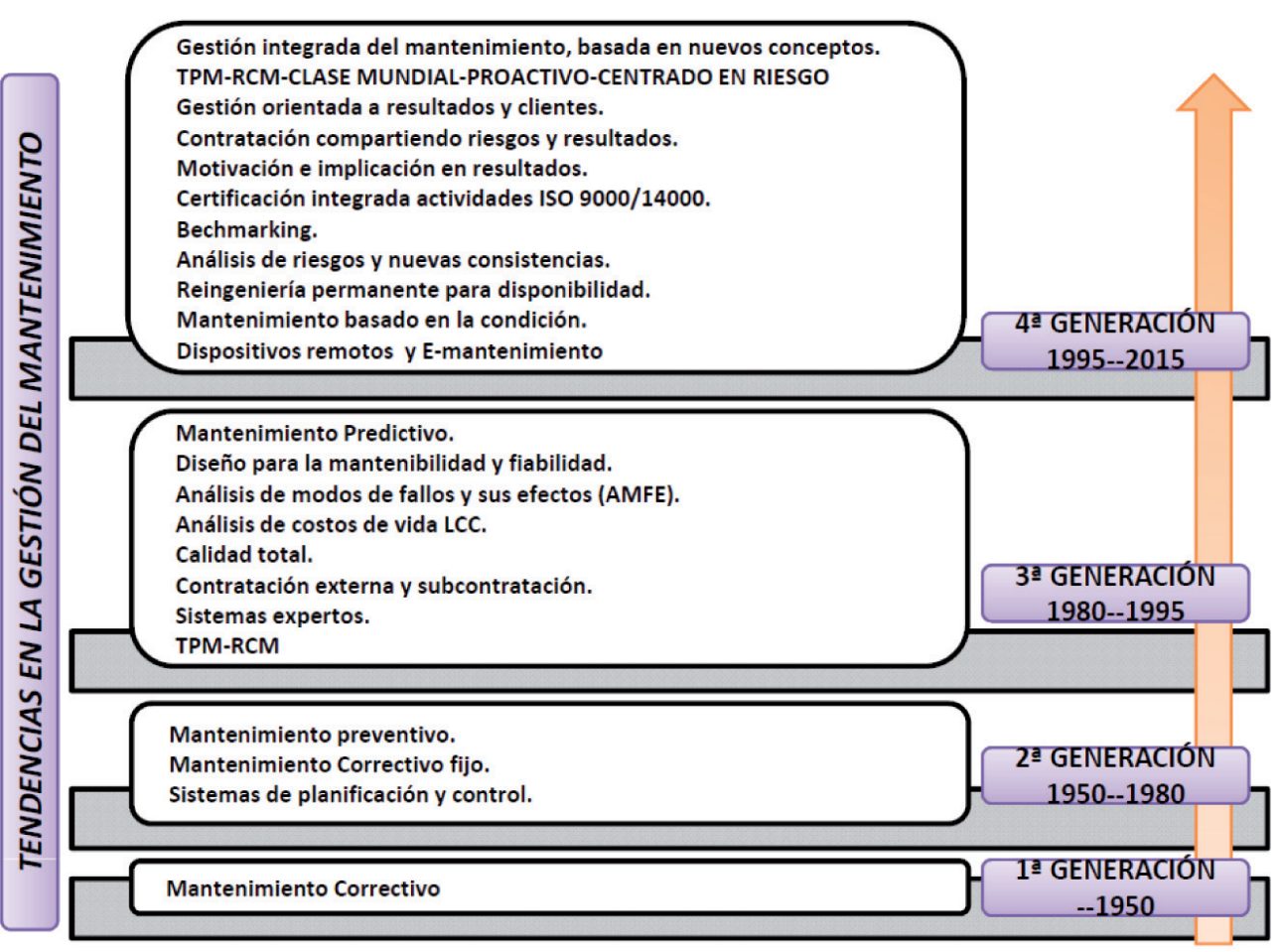

Fig. 5: Tendencias en la gestión del mantenimiento. Fuente: Elaboración propia

\section{CONCLUSIONES}

En la propia evolución de las empresas y dentro de ellas la actividad de mantenimiento a través de la historia se observan factores bien definidos por Maslow [40], donde se ha ido evolucionando en referencia al conocimiento desde la mera supervivencia hasta los conceptos de calidad total y estudio del ciclo de vida de los equipos e instalaciones (Figura 6). En el artículo se resume la incidencia y la consecuencia del desarrollo del conocimiento en relación a los factores estratégicos esenciales considerados, y que afectan de manera fundamental en toda la actividad de mantenimiento, y por consiguiente, en la propia empresa.

En un principio eran los propios operarios quienes se encargaban de las reparaciones de los equipos, basándose todo el co-

La creciente importancia de los Sistemas de Gestión de Mantenimiento asistido por Ordenador, Mantenimiento Basado en la Condición, y la aplicación de dispositivos remotos y funciones como el e-mantenimiento, han abierto grandes posibilidades para la optimización del mantenimiento [39], y la mejora de la gestión del conocimiento [42].

Como ya se ha descrito, una vez comprobado cómo, a lo largo de la historia y evolución del mantenimiento industrial, se perfilan los elementos esenciales del mismo, y que precisan dada su intima relación, con los factores que posibilitan una adecuada gestión de la información y conocimiento.

\section{Jerarquía de las necesidades de Maslow}

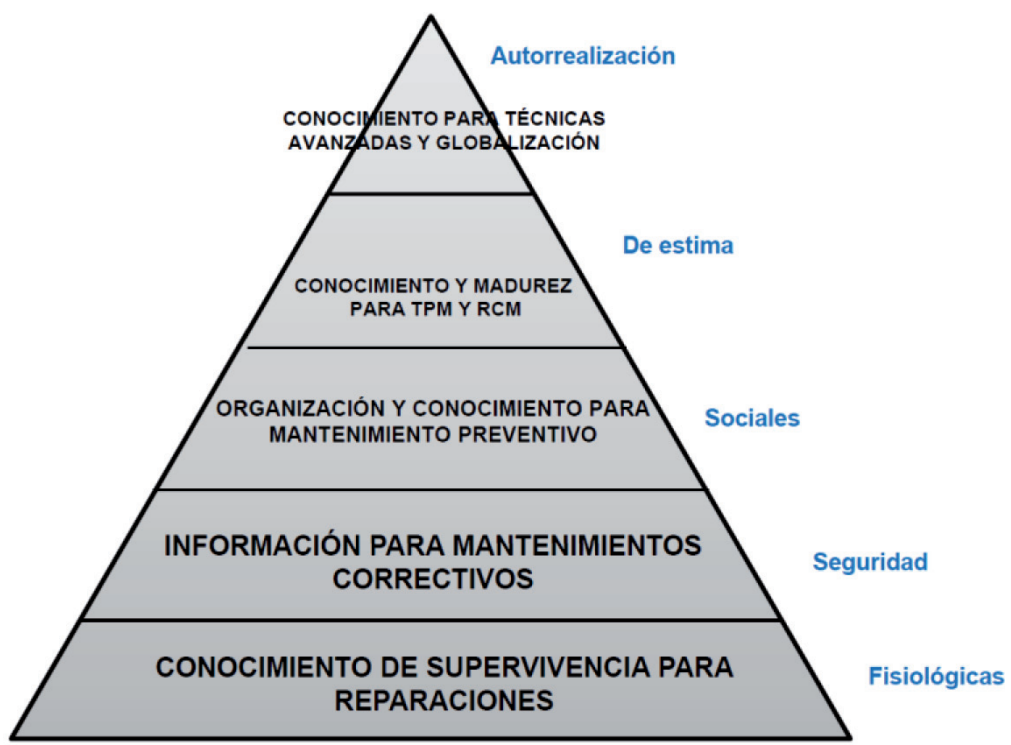

Fig. 6: Evolución del mantenimiento en base a la necesidad de conocimiento. Fuente: Elaboración propia a partir de [40] nocimiento fundamentalmente en tácito basado en las propias experiencias y vivencias. Conforme los sistemas de producción y edificaciones se fueron haciendo más complejas y la dedicación a tareas de reparación aumentaba, empezaron a crearse los primeros departamentos de mantenimiento, con una actividad diferenciada de los operarios de producción, y con un conocimiento más especializado.

Tras la aparición de los conceptos de fiabilidad a partir de la Primera Guerra Mundial y, sobre todo, de la Segunda, los departamentos de mantenimiento buscan no sólo solucionar las averías que se producen en los equipos sino además prevenirlas, actuar para que no se produzcan. Esto supone crear nuevas figuras en los departamentos de mantenimiento, personal cuya función es estudiar qué tareas de mantenimiento deben realizarse para aumentar la eficiencia del servicio, con un conocimiento organizacional más preciso y científico. Con este conocimiento se busca aumentar y fiabilizar la producción, evitar las pérdidas por averías y sus costes asociados. A través de este análisis más profundo y el conocimiento y la experiencia alcanzada, marcan las bases para que aparecen casi sucesivamente diversos métodos de mantenimiento, cada uno aplicado a las necesidades concretas de cada proceso industrial: el Mantenimiento Preventivo (revisiones y limpiezas periódicas), el Mantenimiento Predictivo (análisis del estado de los equipos mediante el análisis de variables físicas), el Mantenimiento Proactivo (implicación del personal en labores de mantenimiento), el mantenimiento productivo total con su relación con los sistemas de calidad total [43], la Gestión de Mantenimiento Asistida por Ordenador, y el Mantenimiento Basado en Fiabilidad. El RCM como estilo de gestión de mantenimiento, se basa en el estudio y conocimiento de los equipos, en 
análisis de los modos de fallo y en la aplicación de técnicas estadísticas y tecnología de detección.

Como se puede comprobar, las diferentes técnicas de mantenimiento han ido evolucionando a lo largo del último siglo en función de las carencias (técnicas y de conocimiento) que se observaban en cada uno de los modelos de mantenimiento al aplicarlos a la situación industrial real, de manera que unas engloban a otras, algunas interactúan entre ellas, y todas se han ido adaptando a la situación práctica de la industria y búsqueda de una mayor eficiencia.

Con todo lo expuesto se fundamenta la necesidad de concienciar a los órganos de dirección de las empresas y de los departamentos de mantenimiento del gran valor estratégico que supone el conocimiento dentro del capital humano que opera en mantenimiento, y que sin duda, pueden influir en la eficiencia global de la empresa.

\section{PARA SABER MÁS}

[1] Carcel-Carrasco, , F. J., Rodriguez-Mendez, M. "INDUSTRIAL MAINTENANCE AND TACIT KNOWLEDGE: AN INTRODUCTION ABOUT ITS INCIDENCE" DYNA Management. Enero-Diciembre 1. (2013):[No Consta]. DOI: http://dx.doi.org/10.6036/MN5857

[2] Del Moral, A. Gestión del Conocimiento. Thompson Editores. España, 2007.

[3] Sánchez,M; Chaminade, C;Escobar, C. En busca de una teoría sobre medición y gestión de los intangibles en la empresa: Una aproximación metodológica. Ekonomiaz, n 45, pp. 188-213, 1999.

[4] Peña Vendrell, Pablo.To know or no to be. Conocimiento, el oro gris de las organizaciones. DINTEL, Madrid, 2001.

[5] OCDE, Medición de la gestión de conocimientos en las empresas: primeros resultados. Ed. OCDE, 2004.

[6] Alsyouf, I. The role of maintenance in improving companies, productivity and profitability. International Journal of Production Economics. Vol. 105 No. 1. pp. 70-78, 2007. . DOl: http://dx.doi. org/10.1016/j.ijpe.2004.06.057

[7] Lopez, P.; Centeno, G. Integrated system to maximize efficiency in transit maintenance departments. International Journal of Productivity and Performance Management. Vol. 55 No. 8.. pp. 638-654, 2006. DOI: http://dx.doi.org/10.1108/17410400610710189

[8] Kans, M. The advancement of maintenance information technology A literature review. Journal of Quality in Maintenance Engineering. Vol. 15 No. 1,. pp. 5-16, 2009. . DOI: http://dx.doi. org/10.1108/13552510910943859

[9] Basim Al-Najjar and Mirka Kans. A model to identify relevant data for problem tracing and maintenance cost-effective decisions. A case study. International Journal of Productivity and Performance Management. Vol. 55 No. 8, pp. 616-637, 2006. DOl: http://dx.doi. org/10.1108/17410400610710170

[10] Peluffo, M., Catalán, E. Introducción a la gestión del conocimiento y su aplicación al sector publico. Ed. Instituto Latinoamericano y del Caribe de Planificación, 2002.

[11] Nonaka, I., Konno, N. "The concept of "Ba": building a foundation for knowledge creation", California Management Review, vol. 40, n 3, Spring, pp. 40-54, 1998.

[12] Xiomara, P. La gestión del conocimiento y las Tics en el siglo XXI. CONHISREMI, Revista Universitaria de Investigación y Diálogo Académico, Vol. 5, No. 1. 2009.

[13] Nonaka, I., Takeuchi, H. La Organización Creadora de Conocimiento. Oxford. México, 1999.

[14] Greer, R.W., "Records Make the Difference", Coal Age. Vol. 65, 1960.

[15] Hanks, H., "Program for Maintenance of Mobile Equipment", Min. Congress Joum. Vol. 47, № 9, pp.35-38, 1961.

[16] Barlow, R. E., Hunter, L. C., \& Proschan, F. Optimum checking procedures. Journal of the Society for Industrial \&t Applied Mathematics, 11(4), 1078-1095. 1963

[17] Darnell, H., Bert, A., "The role of maintenance management in achieving industrial efficiency", Maintenance'78. BMCA, Londres, 1978.

[18] Christer, T., "Ways of assessing and improving maintenance performance". PEMEC'81, Birmingham, 1981.

[19] Boland, P.J.; Proscan, F., "Periodic Replacement with Increasing Minimal Repair Cost at Failure", Operations Research, 30, pp. 1083-1089, 1982.
[20] British Standars, Glossary of General Terms used in maintenance Organization. (BS 3811:1964), 1964.

[21] Kelly, A.; Harris, M.J., "Management of Industrial Maintenance", Butterworths, Oxford, 1978.

[22] Buttery, L.M., "New survey of U.S. Maintenance Costs. Hydrocarbon.", Processing, Jan. 1978.

[23] Crespo, A. Ingeniería de mantenimiento. Técnicas y métodos de aplicación a la fase operativa de los equipos. AENOR. Madrid, 2004.

[24] López Campos M, Fumagalli L, Gómez Fernández J, Crespo Márquez A, Macchi M. UML model for integration between RCM and CBM in an e-maintenance architecture. Proceedings of the First IFAC Workshop on Advanced Maintenance Engineering Services and Technology (A-MEST), Lisbon, Portugal, pp. 133--138, 2010.

[25] Muller A, Crespo Márquez A, lung B. On the concept of e-maintenance: Review and current research. Reliability Engineering and System Safety 2008; 93:pp.1165--1187, 2008. . DOl: http://dx.doi.org/10.1016/j. ress.2007.08.006

[26] Reiner J, Koch J, Krebs I, Schnabel S, Siech T. Knowledge management issues for maintenance of automated production systems. Proceedings of the IFIP International Conference on Human Aspects in Production Management, Karlsruhe, Germany, vol. 160, pp. 229--237,2005.

[27] Moubray, J. Reliability-Centered Maintenance", ButterworthHeinemann, Oxford, 1991.

[28] Smith, M. - Reliability Centered Maintenance - McGraw Hill, Inc. School Education Group - New York - USA. 1992.

[29] Nakajima, S. Introduction to TPM", Productivity Press, Cambridge, MA. 1988.

[30] Cárcel Carrasco, F.J.; Grau, J. Aspectos estratégicos del mantenimiento industrial relativos a la eficiencia energética, Revista Mantenimiento. № 257, Septiembre. pp 25-30, 2012.

[31] Oiltech Analisys S.L. Mantenimiento Proactivo de sistemas mecánicos lubricados - Fluidos oleohidráulica neumática y automación -Vol. 24 Número 208 y 209 - España, 1995.

[32] Pirret, R. Proactive calibration helps drive productivity higher - IECCS. Everett, WA - USA - June, 1999.

[33] Idhammar, C. Maintenance management: moving from reactive to results-oriented - Journal Review Pima's Papermaker -- USA - July, 1997.

[34] Hiatt, B. Best Practices Maintenance - A 13 Step Program in Establishing a World Class Maintenance Organization - USA. 1999.

[35] Serratella CM, Wang G, Conachey R. Risk-based strategies for the next generation of maintenance and inspection programs. International Symposium on Maritime, Safety, Security and Environmental Protection (SSE), Athens, Greece, pp. 20-21, 2007.

[36] Turan, 0., Lazakis, l., Judahb, S., Incecika, A... Investigating the Reliability and Criticality of the Maintenance Characteristics of a Diving Support Vessel. Qual. Reliab. Engng. Int., 27,pp. 931-946, 2011. DOI: http://dx.doi. org/10.1002/qre.1182.

[37] Zaphiropoulos EP, Dialynas EN. Methodology for the optimal component selection of electronic devices under reliability and cost constraints. Quality Reliability Engineering International, 23, pp.885897, 2007. DOI: http://dx.doi.org/10.1002/qre.850

[38] Lazakis I, Turan O, Aksu S. Increasing ship operational reliability through the implementation of a holistic maintenance management strategy. Journal of Ships and Offshore Structures, 5(4),pp.337--357, 2010. DOI:1 $0.1080 / 17445302.2010 .480899$

[39] Jonsson P, "The status of maintenance management in Swedish manufacturing firms", Journal of Quality in Maintenance Engineering, Vol. 3-4, pp. 233-258. 1997. DOI: 10.1108/13552519710176863.

[40] Maslow, A. A Theory of Human Motivation. Originally Published in Psychological Review, 50, pp. 370-396, 1943.

[41] Conde, J. "El Mantenimiento efectivo: principios y métodos". Working paper, GI0-0500-UCLM, Ciudad Real, 1999.

[42] Cárcel Carrasco, Francisco Javier. "Planteamiento de un modelo de mantenimiento industrial basado en técnicas de gestión del conocimiento". Omnia Science, 2014. DOI: http://dx.doi.org/10.3926/ oms.198.

[43] Cárcel Carrasco, Francisco Javier. "La gestión del conocimiento en la ingeniería del mantenimiento industrial: Investigación sobre la incidencia en sus actividades estratégicas". Omnia Science, 2014. ISSN 978-84-941872-7-8. DOl: http://dx.doi.org/10.3926/oms.197.

[44] Matteson, Thomas D. Airline experience with reliability-centered maintenance. Nuclear Engineering and Design, vol. 89, no 2, p. 385-390. 1985. doi:10.1016/0029-5493(85)90076-7.

[45] Ackert, Shannon P. Basics of aircraft maintenance programs for financiers-evaluation \&t insights of commercial aircraft maintenance programs.Online. www. aircraftmonitor. com/ uploads/1/5/9/9/15993320/basics_of_aircraft_maintenance_programs_ for_financiers_v1. pdf (accessed 14 January 2016), 2010.

[46] UNE EN 13306.Aenor. Mantenimiento. Terminología del mantenimiento. Marzo de 2011 